\title{
Role of intensive nutrition support and prophylactic percutaneous endoscopic gastrostomy during concomitant chemoradiotherapy for oropharyngeal cancer
}

\author{
Miki Takahashi ${ }^{1}$. Nana Kosaka ${ }^{5}$ - Emi Wakui ${ }^{1}$ Shinobu Iwaki ${ }^{1}$ Mika Nishii ${ }^{1}$ Masanori Teshima ${ }^{2}$. \\ Hirotaka Shinomiya ${ }^{2} \cdot K_{\text {Koichi Morimoto }}{ }^{2}$ Naomi Kiyota $^{3} \cdot$ Ryohei Sasaki $^{4} \cdot$ Makoto Usami $^{5} \cdot$ Naoki Otsuki $^{2}$. \\ Ken-ichi Nibu ${ }^{2}$ (D)
}

Received: 16 May 2018 / Accepted: 31 July 2018 / Published online: 18 August 2018

(c) The Author(s) 2018

\begin{abstract}
Background Concomitant chemoradiotherapy (CCRT) produces severe mucositis and swallowing dysfunction, often resulting in malnutrition. Intensive nutrition support (INS) in addition to percutaneous endoscopic gastrostomy (PEG) is reported to decrease adverse effects during CCRT.

Patients and Methods Fifty-eight patients with oropharyngeal cancer treated by CDDP-based CCRT were retrospectively analyzed. Twenty-nine patients treated with INS in addition to PEG were classified as INS group, and other 29 patients treated with PEG but without INS were classified as control group.

Results INS in addition to PEG significantly increased calorie intake in the second half of CCRT and reduced adverse events including mucositis $(p=0.0019)$, leukopenia $(p=0.04)$, and renal function $(p=0.006)$. Moreover, 21 out of 29 patients had successfully administration of $200 \mathrm{mg} / \mathrm{m} 2$ or more of CDDP, while only 10 out of 29 patients had enough amount of CDDP in control group.

Conclusions These results suggest that INS in addition to prophylactic PEG not only decreases adverse effects but also may potentially improve oncological outcome of the patients with oropharyngeal cancer treated by CCRT.
\end{abstract}

Keywords Percutaneous endoscopic gastrostomy $\cdot$ Intensive nutrition support · Oropharyngeal cancer $\cdot$ Chemoradiotherapy

\section{Introduction}

To preserve the swallowing and verbal communication function, platinum-based concurrent chemoradiotherapy has been employed as the standard of care for the advanced

Naoki Otsuki

naokies@med.kobe-u.ac.jp

1 Department of Rehabilitation, Kobe University Hospital, Kobe, Japan

2 Department of Otolaryngology-Head and Neck Surgery, Kobe University Hospital, Kobe, Japan

3 Department of Medical Oncology and Hematology, Kobe University Hospital, Kobe, Japan

4 Department of Radiation Oncology, Kobe University Hospital, Kobe, Japan

5 Division of Nutrition and Metabolism, Kobe University Graduate School of Health Sciences, Kobe, Japan pharyngeal and laryngeal cancers to improve the organ preservation and survival rates as well as quality of life after the treatment of head-and-neck cancers [1, 2]. However, CCRT for head-and-neck cancers produces severe mucositis, intolerable pain, and swallowing dysfunction, often resulting in a significant malnutrition, especially when oropharynx is included in the radiation field [3, 4]. European Society for Clinical Nutrition and Metabolism (ESPEN) guidelines stated that malnutrition during the treatment of cancers decreases quality of life and activity of daily life, increases side effects, and decreases response to treatments, as a result, survival periods of the patients [5]. According to the recent Cochrane review, weight loss 6 months after the initiation of radiotherapy or chemotherapy was significantly lower in the patients with nasogastric tube in comparison with the patients without gastric tube [6]. On the other hand, in the randomized study intended for the patients treated by radiotherapy as outpatient care, active nutrition support had 
significantly prevented weight loss and maintained quality of life [7].

Based on this background, from Sept. 2007, we started to recommend percutaneous endoscopic gastrostomy (PEG) to our patients before CCRT for oropharyngeal cancer [8]. However, in spite of PEG placement, average daily intake of calories of the patients was revealed as $20.2 \pm 11.7 \mathrm{kcal} /$ $\mathrm{kg} / \mathrm{day}$, which was far less than recommended by ESPEN. Thus, from Dec. 2010, we started intensive nutrition support (INS) by head-and-neck nutrition support team (H\&N NST) consisting of head-and-neck surgeons, ward nursing stuff, ward pharmacist, managerial dietician, speech therapist, and dental hygienist for these patients with PEG during CCRT for oropharyngeal cancer to maintain total intake of calories at $30-35 \mathrm{kcal} / \mathrm{kg} / \mathrm{day}$. In this study, we retrospectively investigated the effectiveness of INS in the patients with PEG during CCRT for oropharyngeal cancer to maintain total intake of calories at $30-35 \mathrm{kcal} / \mathrm{kg} / \mathrm{day}$.

\section{Patients and methods}

Fifty-eight patients with oropharyngeal cancer treated by CDDP-based CCRT as an initial treatment with curative intent between 2007 and 2013 at the department of Otolaryngology-Head and Neck Surgery, Kobe University Hospital were enrolled in this study. All the patients had PEG placement before starting CCRT. Prior to PEG placement, patients had gastroscopic examination and computed tomography of abdomen to rule out the contraindication for PEG, and informed consent was achieved from all patients.

Patients treated with PEG and INS between 2007 and 2013 were retrospectively classified as INS group, and patients treated with PEG but without INS between Sept. 2007 and Nov. 2010 were classified as control group. Irradiation was given at $2 \mathrm{~Gy} /$ fraction $\mathrm{x} 5$ days/week. In general, a total of 40-50Gy was irradiated at prophylactic field and a total of approximate 70 Gy was irradiated at primary site and metastatic lymph nodes by three-dimensional (3D) conformal radiation therapy during the observation period. Principally, $80 \mathrm{mg} / \mathrm{m}^{2}$ of CDDP was administrated every triweekly. However, amount of CDDP was adjusted in each patient considering renal function, myelosuppression, and general condition. As anti-emetic agents, granisetron hydrochloride, metoclopramide, and ramosetron hydrochloride were used in control group, and granisetron hydrochloride, palonosetron hydrochloride, and aprepitant were used in INS group according to the recommendation made by the institutional review board for medical oncology. To prevent swallowing disorders, all the patients of both groups had swallowing rehabilitation by speech therapists as well as oral care by dental hygienists during and after CCRT.
Patients' data including age, sex, height, weight, subsite of oropharyngeal cancer, stage, TNM classification according to UICC 7th TM classification, doses of CDDP and irradiation, intake of calories, use of PEG tube, laboratory data, and adverse event during CCRT were retrospectively collected from medical records. Adverse events during CCRT, including myelosuppression, mucositis, nausea, vomiting, complications of PEG, and requiring break or dose reduction of radiation and/or CDDP, were evaluated. Myelosuppression was evaluated as the numbers of white blood cells (WBC) and lymphocyte. Adverse events were classified according to the Common Terminology Criteria for Adverse Events ver. 4.0 (CTCAE) [9]. Nutrition status was evaluated as daily intake of calories, weight, body mass index [BMI: weight $(\mathrm{kg}) /$ height $\times$ height $\left(\mathrm{m}^{2}\right)$ ], and serum albumin level (Alb). Modes of obtaining nourishments were classified as follows: oral intake (Oral), enteral nutrition via PEG tube (EN), and parenteral nutrition (PN). Adverse events and nutrition status were evaluated at the beginning of CCRT, $20 \mathrm{~Gy}, 40 \mathrm{~Gy}$, and $60 \mathrm{~Gy}$ of irradiation, and the end of CCRT. Body weight change rate was determined as weight at the end of CCRT divided by weight at the beginning of CCRT.

Radiotherapy was determined as "completion" if scheduled dose of radiation was fully administrated without break and chemotherapy was determined as "completion" if a total of $200 \mathrm{mg} / \mathrm{m}^{2} \times$ body surface area (BSA) or more of CDDP were administrated. Statistical analysis including Chi-square test, $t$ test, and Mann-Whitney $U$ test were performed using Microsoft Excel and its add-in soft Statcel 2 (Seiun-sha, Tokyo). Survival was estimated by the Kaplan-Meier method and log-rank test was used for statistical analysis for survival. $P$ value less than 0.05 was determined as significant. This study was conducted as the collaboration study of departments of Otolaryngology-Head and Neck Surgery, Rehabilitation and Nutrition Support of Kobe University Hospital and Kobe University Graduate School of Health Science. All the procedures in this study were approved by Kobe University Ethical Committee (Approved Number: 1589 ) and written informed consents were observed in all patients.

\section{Results}

\section{Background of the patients}

Characteristics of the patients of INS and control groups are shown in Table 1. No significant differences were observed between the two groups in age, sex, weight, BMI, subsite, stage, T classification, and duration of CCRT. 
Table 1 Characteristics of the patients

\begin{tabular}{|c|c|c|c|}
\hline Characteristics & Control & INS & $P$ Value \\
\hline Age, mean(SD), year & $62.1 \pm 8.0$ & $59.7 \pm 9.7$ & 0.31 \\
\hline Male/female & $22 / 7$ & $25 / 4$ & 0.31 \\
\hline Weight (kg) & $61.8 \pm 12.1$ & $59.6 \pm 13.0$ & 0.50 \\
\hline BMI $\left(\mathrm{kg} / \mathrm{m}^{2}\right)$ & $22.6 \pm 3.2$ & $21.6 \pm 3.7$ & 0.26 \\
\hline Creatinine $(\mathrm{mg} / \mathrm{dl})$ & $0.86 \pm 0.20$ & $0.81 \pm 0.17$ & 0.31 \\
\hline $\mathrm{eGFR}\left(\mathrm{ml} / \mathrm{min} / 1.73 \mathrm{~m}^{2}\right)$ & $68.3 \pm 13.9$ & $75.8 \pm 16.8$ & 0.07 \\
\hline Smoker/nonsmoker & $16 / 13$ & $18 / 11$ & 0.59 \\
\hline Radiation dose(Gy) & $69.1 \pm 2.2$ & $69.9 \pm 0.7$ & 0.09 \\
\hline Performance status & & & 0.07 \\
\hline 0 & $27(93)$ & $19(66)$ & \\
\hline 1 & $1(3)$ & $6(21)$ & \\
\hline 2 & $0(0)$ & $1(3)$ & \\
\hline 3 & $1(3)$ & $3(10)$ & \\
\hline Primary site of tumor & & & 0.11 \\
\hline Lateral & $21(70)$ & $18(62)$ & \\
\hline Anterior wall & $7(23)$ & $4(13)$ & \\
\hline Superior wall & $1(3)$ & $3(10)$ & \\
\hline Posterior wall & $0(0)$ & $4(13)$ & \\
\hline T stage & & & 0.86 \\
\hline $\mathrm{T} 1$ & $2(7)$ & $3(10)$ & \\
\hline $\mathrm{T} 2$ & $15(52)$ & $17(59)$ & \\
\hline $\mathrm{T} 3$ & $8(28)$ & $6(21)$ & \\
\hline $\mathrm{T} 4$ & $4(14)$ & $3(10)$ & \\
\hline $\mathrm{N}$ stage & & & 0.41 \\
\hline 0 & $3(10)$ & $4(14)$ & \\
\hline 1 & $3(10)$ & $4(14)$ & \\
\hline $2 \mathrm{a}$ & $2(7)$ & $7(24)$ & \\
\hline $2 b$ & $13(45)$ & $10(35)$ & \\
\hline $2 c$ & $7(24)$ & $3(10)$ & \\
\hline 3 & $1(3)$ & $1(3)$ & \\
\hline Stage & & & 0.17 \\
\hline II & $0(0)$ & $4(14)$ & \\
\hline III & $5(17)$ & $3(10)$ & \\
\hline IVA & $23(80)$ & $20(69)$ & \\
\hline IVB & $1(3)$ & $1(7)$ & \\
\hline
\end{tabular}

Control control group, INS intensive nutrition support group, BMI body mass index, $e G F R$ estimated glomerular filtration rate

\section{Amount of daily calorie intake}

As shown in Fig. 1, amount of total daily calorie intake increased during CCRT gradually in both groups. Average amount of total daily calorie intake during the whole treatment period was $20.3 \pm 11.6 \mathrm{kcal} / \mathrm{kg} /$ day in control group, and $24.8 \pm 7.2 \mathrm{kcal} / \mathrm{kg} / \mathrm{day}$ in INS group, respectively $(P=$ 0.08). As CCRT went on, amount of oral intake decreased, while EN intake increased in both groups (Fig. 2a, b). Parenteral nutrition was administrated via peripheral solution

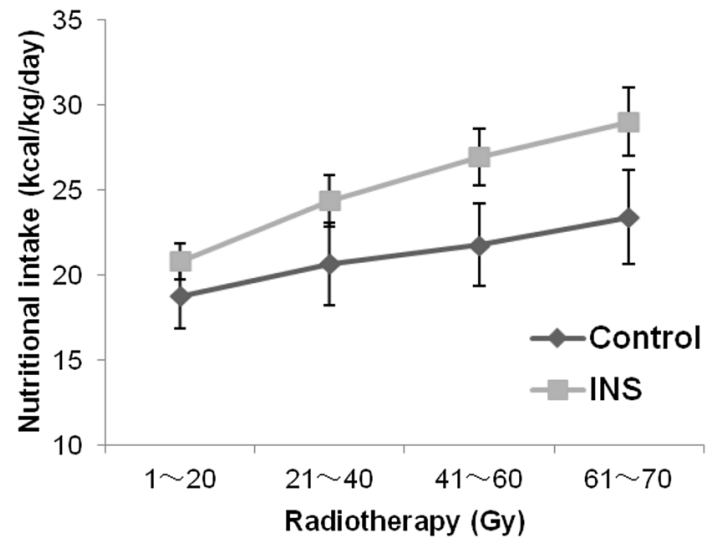

Fig. 1 Changes in nutritional intake during concurrent chemoradiotherapy. Control: control group, INS: intensive nutrition support group

as required. No patient had central venous hyperalimentation neither in INS group nor control group.

The number of patients who took in more than $25 \mathrm{kcal} / \mathrm{kg} /$ day was 5 at start, 7 at $20 \mathrm{~Gy}, 6$ at $40 \mathrm{~Gy}, 9$ at $60 \mathrm{~Gy}$, and 5 in average in control group, while it was 8 at start, 12 at $20 \mathrm{~Gy}$, 15 at $40 \mathrm{~Gy}, 18$ at $60 \mathrm{~Gy}$, and 12 in average in INS group. The number of patients was significantly higher in INS group at $40 \mathrm{~Gy}, 60 \mathrm{~Gy}$, and in average $(P=0.04, P=0.01$, $P=0.02)$. Body weights and BMI significantly decreased in both groups $(P<0.001)$. There were no significant differences in the ratio of body weight loss (Fig. 3a) or BMI loss (Fig. 3b) between the two groups.

\section{Adverse effect during CCRT}

Adverse effects during CCRT are summarized in Table 2. Mucositis at the end of CCRT and leukopenia at $60 \mathrm{~Gy}$ were significantly milder in INS group in comparison with control group ( $P=0.0019$ and 0.04 , respectively). While no significant difference was observed in albumin level or eGFR at $60 \mathrm{~Gy}$ in terms of CTCAE grading system, albumin level count at 60 Gy tended to be higher in INS group in comparison with control group $(3.5 \pm 0.4$ vs. $3.3 \pm 0.4 \mathrm{~g} / \mathrm{dl}, P=0.06)$ and actual value of eGFR was significantly higher in INS group in comparison with control group $(72.4 \pm 17.9$ vs. $\left.59.8 \pm 16.1 \mathrm{ml} / \mathrm{min} / 1.73 \mathrm{~m}^{2}, P=0.006\right)$. eGFR significantly decreased during CCRT in control group $(P=0.001)$, but not in INS group. Among the INS group, the average daily calorie intake of the patients in whom CTCAE grades of WBC counts, lymphocyte counts, and serum albumin level at the end of CCRT were grade 2 or less $(n=12)$ was significantly higher than those of other patients $(28.05 \pm 7.2 \mathrm{kcal} / \mathrm{kg} /$ day $(n=12)$ vs. $22.5 \pm 6.1 \mathrm{kcal} / \mathrm{kg} / \mathrm{day}(n=17), P=0.04)$. 


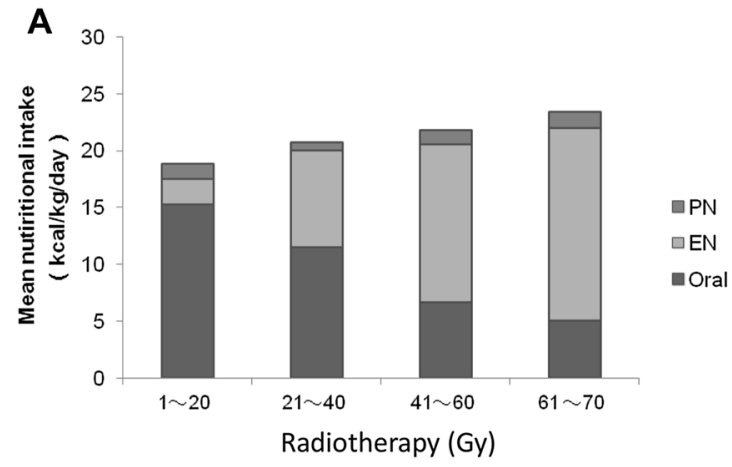

Fig. 2 Changes in nutritional Intake according to the nourishment modes. a Control group, b intensive nutrition support group. $P N$ mean calories (kcal/kg/day) of parenteral nutrition, $E N$ mean calories

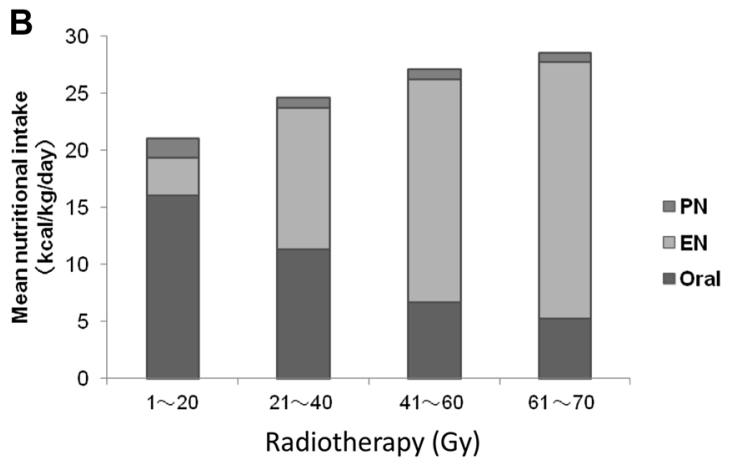

(kcal/kg/day) of enteral nutrition via PEG, and Oral mean calories $(\mathrm{kcal} / \mathrm{kg} /$ day $)$ of oral intake
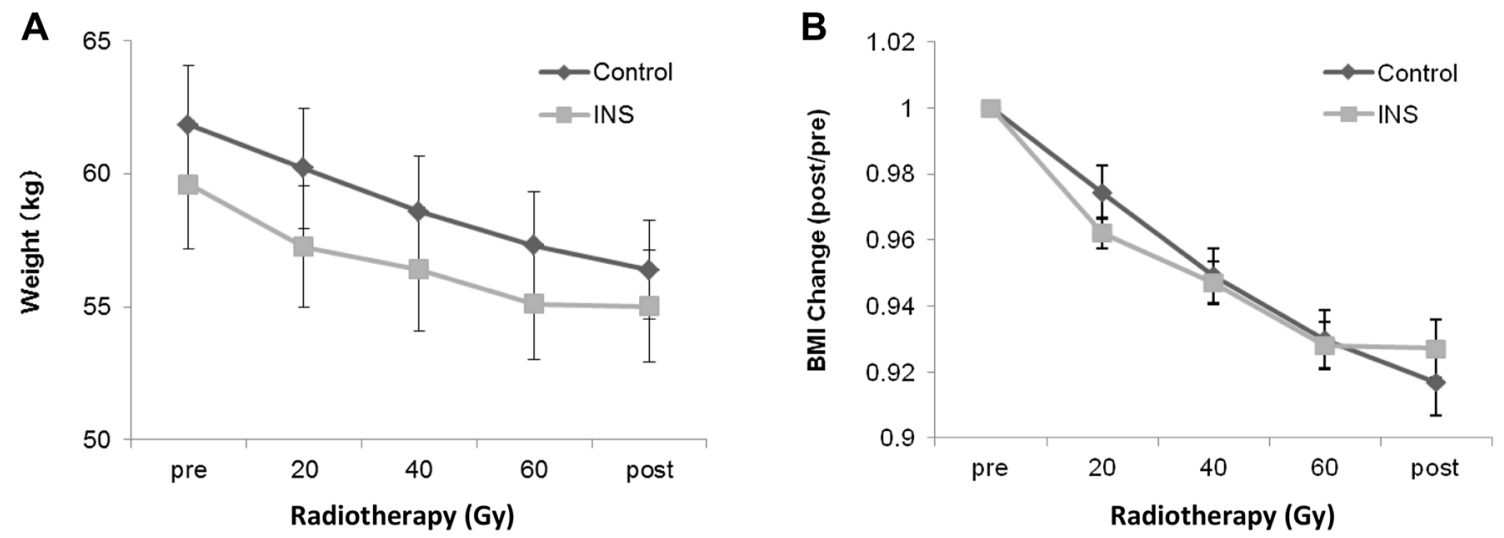

Fig. 3 Changes in body weight and body mass index during concurrent chemoradiotherapy. a Body weight; b body mass index. Control control group, INS intensive nutrition support group, $B M I$ body mass index, $C D D P$ cisplatin, and $R T$ radiation

\section{Completion rates of concomitant chemoradiotherapy and overall survival rates}

In both groups, 28 of 29 patients (97\%) underwent scheduled dose of radiation (approximate $70 \mathrm{~Gy}$ ) without break. Irradiation was terminated at $60 \mathrm{~Gy}$ in one patient of control group and $66 \mathrm{~Gy}$ in another patient of INS group due to aspiration pneumonia. In terms of concomitant chemotherapy, a total of $200 \mathrm{mg} / \mathrm{m}^{2} \times$ BSA or more of CDDP were administrated in 21 patients $(72 \%)$ of INS group, but in only 8 patients (28\%) of control group $(P=0.0038$, Table 3). 5-year overall survival rates of control group and INS group were 88.7 and $83.6 \%$, respectively $(p=0.55)$.

\section{Duration of PEG Tube}

Principally, PEG tube was decannulated after confirming the complete remission by positron emission tomography/computed tomography (PET-CT) 3 months after the completion of CCRT. PEG tube was decannulated in 22 patients of control group (76\%) and 20 patients (69\%) in INS group. Median duration of PEG tube inserted was 130.4 days (37-490) in INS group and 134 days (10-504) in control group.

\section{Discussion}

In the present study, the number of the patients who took more than $25 \mathrm{kcal} / \mathrm{kg} /$ day was significantly in INS group at 40 and $60 \mathrm{~Gy}$, reflecting that calories were intensively administrated by EN in INS group in comparison with control group during the second half of CCRT. However, the average amount of calorie intake during the whole period of CCRT failed to reach target not only in control group but also in INS group. Despite of PEG and intensive nutrition support, patients significantly lost their weight not only in control group but also in INS group, in contrast with the previous report [5, 10-13]. Of note, patients of 
Table 2 Adverse effect during concurrent chemoradiotherapy according to CTCAE

\begin{tabular}{|c|c|c|c|c|}
\hline & Grade & Control & INS & $P$ Value \\
\hline \multirow[t]{5}{*}{ Mucositis at the end of CCRT } & & & & 0.0019 \\
\hline & 1 & $0(0)$ & $3(10)$ & \\
\hline & 2 & $5(17)$ & $13(45)$ & \\
\hline & 3 & $19(66)$ & $13(45)$ & \\
\hline & 4 & $5(17)$ & $0(0)$ & \\
\hline \multirow[t]{3}{*}{ Albumin level at $60 \mathrm{~Gy}$} & & & & 0.47 \\
\hline & 1 & $23(82)$ & $27(93)$ & \\
\hline & 2 & $5(18)$ & $2(7)$ & \\
\hline \multirow[t]{4}{*}{ Leukopenia at $60 \mathrm{~Gy}$} & & & & 0.04 \\
\hline & 1 & $14(48)$ & $23(80)$ & \\
\hline & 2 & $12(41)$ & $5(17)$ & \\
\hline & 3 & $3(10)$ & $1(3)$ & \\
\hline \multirow[t]{5}{*}{ Lymphopenia at $60 \mathrm{~Gy}$} & & & & 0.09 \\
\hline & 1 & $0(0)$ & $1(3)$ & \\
\hline & 2 & $5(17)$ & $10(35)$ & \\
\hline & 3 & $22(76)$ & $18(62)$ & \\
\hline & 4 & $2(7)$ & $0(0)$ & \\
\hline \multirow[t]{3}{*}{ Creatinine at the end of CCRT } & & & & 0.82 \\
\hline & 1 & $27(93)$ & $26(90)$ & \\
\hline & 2 & $2(7)$ & $3(10)$ & \\
\hline \multirow[t]{4}{*}{ eGFR at the end of CCRT } & & & & 0.66 \\
\hline & 0 & $26(90)$ & $28(97)$ & \\
\hline & 1 & $2(7)$ & $0(0)$ & \\
\hline & 2 & $1(3)$ & $1(3)$ & \\
\hline
\end{tabular}

CTCAE Common Terminology Criteria for Adverse Events ver.4.0, INS intensive nutrition support group, $e G F R$ estimated glomerular filtration rate, $C C R T$ concurrent chemoradiotherapy

Table 3 Completion rates of treatment

\begin{tabular}{lll}
\hline & Control & INS \\
\hline $\begin{array}{l}\text { Average duration (day) } \\
\text { Completion rates of RT }\end{array}$ & 50.03 & 53.1 \\
Concomitant chemotherapy & $28 / 29(97)$ & $28 / 29(97)$ \\
$\geqq$ CDDP $200 \mathrm{mg} / \mathrm{m}^{2}(\mathrm{~N})$ & $10(35)$ & $21(72)$ \\
$<\mathrm{CDDP} 200 \mathrm{mg} / \mathrm{m}^{2}(\mathrm{~N})$ & $19(66)$ & $8(28)$ \\
\hline
\end{tabular}

INS intensive nutrition support, $C C R T$ concurrent chemoradiotherapy

INS group lost their weight mostly during the first half of CCRT, while they lost only $0.6 \mathrm{Kg}$ in average during the second half of CCRT. One of the possible reasons was that patients tended to refuse to take calories via PEG tube during the first half of CCRT, even though we recommended EN. These findings suggested the necessity of patient education to make them understand the importance of calorie administration via PEG tube from the beginning of the CCRT. In addition, calories were consistently administrated via PN through the CCRT period in both groups.
Meticulous nutrition management using PN in addition to $\mathrm{OE}$ and EN in view of the patient's condition such as nausea and/or vomiting due to CDDP should be considered.

In terms of the adverse events, mucositis, leukopenia, and lymphopenia at $60 \mathrm{~Gy}$ were milder in INS group in comparison with control group. In addition, among the INS group, WBC and lymphocyte counts and serum albumin level of the patients who had $25 \mathrm{kcal} / \mathrm{kg} /$ day or more, in average, were significantly better than those of the patients who had less. The average daily calorie intake of the patients in whom CTCAE grades of WBC counts, lymphocyte counts, and serum albumin level at the end of CCRT were grade 2 was $28 \mathrm{kcal} / \mathrm{kg} / \mathrm{day}$. Taken together, these findings suggested that intensive nutrition support had succeeded to administrate more calories in INS group, resulted in the reduction of adverse events including leukopenia, lymphopenia, and mucositis due to CCRT in accordance with the previous report [13].

Finally, as recently reported $[14,15]$, most patients had administration of CDDP at a total dose of $200 \mathrm{mg} / \mathrm{m}^{2} \mathrm{x}$ BSA or more in INS group but did not in control group. As above mentioned, renal function in terms of actual value of eGFR was significantly better through the CCRT period in INS group, which might enable to administrate the enough amount of CDDP. Although no significant difference was observed in the survival rates in the present study, administration of $200 \mathrm{mg} / \mathrm{m} 2$ or more of CDDP during CDDP-based CCRT has been reported to be associated with favorable oncological outcome. Thus, the present study suggested that the intensive nutrition support may contribute to improve the oncological outcome of the patients with OPC.

Long-term PEG tube dependence is one of the most critical issues of the prophylactic PEG in the treatment of patients with head-and-neck cancer [16]. Indeed, PEG tube was decannulated in 42 patients but not in other patients at the end of the observation period. Although most common reason of long-term use of PEG tube in the present study was persistent disease, reactive PEG tube placement might be considered as an alternative option in the treatment of oropharyngeal cancer by CCRT, instead of prophylactic PEG. From the other point of view, there was no significant difference in the duration of PEG placement and the number of long-term PEG-dependent patients between INS and control groups, while most patients of INS group tended to start EN intake via PEG earlier by the recommendation of H\&N NST, suggesting the usefulness of swallowing rehabilitation during and after CCRT by speech therapists on the prevention of swallowing disorders after CCRT for oropharyngeal cancer.

One of the limitations of this study was a retrospective study consisting of limited number of the patients. The changed combination of anti-emetic agents might have affected the favorable results of INS group. In terms of the oncological outcome, p16 status of OPC was not evaluated 
in the present study. Another limitation of this study was the lack of subjective data to measure the quality of life of the patients. Since the importance of intensive nutrition support is obvious, randomized study may not be ethically acceptable. Further multi-institute study consisting of large number of patients should be considered to improve the nutrition support during CCRT for the treatment of patients with OPC.

\section{Conclusions}

The present study demonstrated that INS in addition to PEG placement increased the calorie intake and decreased the adverse effects during the CCRT. While INS in addition to PEG placement did not decrease treatment period or weight loss, INS in addition to PEG placement significantly contributed to administrating sufficient amount of CDDP. These results suggest that INS in addition to PEG placement not only decreases the adverse effects during CCRT but also potentially improves oncological outcome of the patients treated by CCRT.

Acknowledgements The authors expressed deep thanks to the former and present nurse managers Ms. Takuko Kunieda and Ms. Eiko Hiroko as well as all the ward nursing staff of Kobe University Hospital, for their great support to the treatment of patients with head-and-neck cancer. This research is partially supported by the grant from Japan Agency for Medical Research and Development (Nibu K, principal investigator, Grant\#: 16ck0106223h001).

\section{Compliance with ethical standards}

Conflict of interest The authors do not have any conflict of interest related to this article to be disclosed.

Open Access This article is distributed under the terms of the Creative Commons Attribution 4.0 International License (http://creativeco mmons.org/licenses/by/4.0/), which permits unrestricted use, distribution, and reproduction in any medium, provided you give appropriate credit to the original author(s) and the source, provide a link to the Creative Commons license, and indicate if changes were made.

\section{References}

1. NCCN Clinical Practice Guidelines in Oncology: Head and Neck Cancers Version 1 (2018) Available via DIALOG https://www. nccn.org/professionals/physician_gls/pdf/head-and-neck.pdf Accessed Apr 2018

2. Adelstein DJ, Li Y, Adams GL et al (2003) An intergroup phase III comparison of standard radiation therapy and two schedules of concurrent chemoradiotherapy in patients with unresectable squamous cell head and neck cancer. J Clin Oncol 21:92-98

3. Nguyen NP, Sallah S (2000) Combined chemotherapy and radiation in the treatment of locally advanced head and neck cancers. In Vivo 14:35-39

4. Cady J (2007) Nutritional support during radiotherapy for head and neck cancer: the role of prophylactic feeding tube placement. Clin J Oncol Nurs 11:875-880

5. Arends J, Bachmann P, Baracos V et al (2017) ESPEN guidelines on nutrition in cancer patients. Clin Nutr 36:11-48

6. Nugent B, Lewis S, O’Sullivan JM (2013) Enteral feeding methods for nutritional management in patients with head and neck cancers being treated with radiotherapy and/or chemotherapy. Cochrane Database Syst Rev

7. Isenring EA, Capra S, Bauer JD (2004) Nutrition intervention is beneficial in oncology outpatients receiving radiotherapy to the gastrointestinal or head and neck area. Br J Cancer 91:447-452

8. Takahashi M, Takemoto N, Sano A et al (2012) Effectiveness of prophylactic percutaneous endoscopic gastrostomy on nutritional status and mucositis in oropharyngeal cancer patients undergoing concurrent chemoradiotherapy. Head and Neck Cancer 38:336342 (in Japanese)

9. Common Terminology Criteria for Adverse Events (CTCAE) Version 4.0 (2009) Available via DIALOG https://www.eortc.be/servi ces/doc/ctc/CTCAE_4.03_2010-06-14_QuickReference_5x7. pdf\#search=\%27CTCAE\%27. Accessed Apr 2018

10. Capuano G, Grosso A, Gentile PC et al (2008) Influence of weight loss on outcomes in patients with head and neck cancer undergoing concomitant chemoradiotherapy. Head Neck 30:503-508

11. Ravasco P, Monteiro-Grillo I, Vidal PM et al (2005) Dietary counseling improves patient outcomes: a prospective, randomized, controlled trial in colorectal cancer patients undergoing radiotherapy. J Clin Oncol 23:1431e8

12. Ravasco P, Monteiro-Grillo I, Marques Vidal P et al (2005) Impact of nutrition on outcome: a prospective randomized controlled trial in patients with head and neck cancer undergoing radiotherapy. Head Neck 27:659e68

13. Langius JA, Zandbergen MC, Eerenstein SE et al (2013) Effect of nutrition therapy on nutritional status, quality of life and mortality in patients with head and neck cancer receiving (chemo) radiotherapy: a systematic review. Clin Nutr 32:671e8

14. Goda M, Jinnouchi O, Takaoka T et al (2015) Efficacy of percutaneous endoscopic gastrostomy on unplanned treatment interruption and nutritional status in patients undergoing chemoradiotherapy for advanced head and neck cancer. J Med Invest 62:173-6

17. Yamazaki T, Enokida T, Wakasugi T et al (2016) Impact of prophylactic percutaneous endoscopic gastrostomy tube placement on treatment tolerance in head and neck cancer patients treated with cetuximab plus radiation. Jpn J Clin Oncol 46:825-831

18. Pohar S, Demarcantonio M, Whiting P et al (2015) Percutaneous endoscopic gastrostomy tube dependence following chemoradiation in head and neck cancer patients. Laryngoscope 125:1366-1371

19. Kramer S, Newcomb M, Hessler J et al (2014) Prophylactic versus reactive PEG tube placement in head and neck cancer. Otolaryngol Head Neck Surg 150:407-412 